\title{
Multiscale Soil Investigations: Physical Concepts and Mathematical Techniques
}

\author{
S. D. Logsdon, E. Perfect, and A. M. Tarquis
} RECENT PUBLICATION on multiscale landscape analysis defined soils as "four dimensional natural bodies
... with the key characteristic of varying with place and time" (Sommer, 2006). Such variation affects how
Wervations are interpreted regarding the evolution, diversity, and dynamics of the soil ecosystem (Heuvelink and
Webster, 2001). Soil variability has often been considered to be composed of "functional" (explained) variations plus
random fluctuations or noise. However, the distinction between these two components is scale dependent because
increasing the scale of observation almost always reveals structure in the noise (Burrough, 1983). Soils can be seen
as the result of spatial variation operating over several scales, indicating that factors influencing spatial variability
differ with scale. This observation points to variability as a key soil attribute that should be studied (Burrough et
al., 1994).

Geostatistical methods and, more recently, fractal-multifractal and wavelet techniques have been used to characterize scaling of soil properties (Zhang et al., 1997; Kravchenko et al., 1999; Eghball et al., 1999). The book edited by Sposito (1998) includes several chapters that discuss various hydrological applications of scaling. Western et al. (2002) reviewed different techniques for scaling soil moisture, including statistical approaches and processbased indices. Hopmans et al. (2002) presented the historical use of scaling in hydrology and discussed the need to extend measurements beyond the laboratory scale to the field or watershed scale. The book by Pachepsky et al. (2003) covers many aspects of scaling and how to reconstruct landscape and watershed processes from small-scale measurements. McBratney et al. (2003) reviewed soil mapping at different scales and approaches for relating soil properties to processes. The group of papers introduced by Pachepsky et al. (2006) dealt with applications of fractal geometry to scaling in soil and related hierarchical systems. Lin et al. (2006) detailed several hypotheses related to the concept of hydropedology to help bridge across disciplines that focus on different ranges of scale. Vereecken et al. (2007) reviewed techniques to upscale soil hydraulic properties, including several "forward" techniques as well as inverse modeling approaches.

An integration of various sources of information and synthesis of diverse approaches is required to study multiscale features that are the product of coexistence, hierarchy, complexity, chaos, and in some cases, self-organization. Understanding the interrelationships between physical, chemical, and biological factors at different scales is essential for research in agriculture, engineering, hydrology, and the environment. The emergence of a more holistic approach to soil science may facilitate a better understanding of both temporal and spatial variability. Concurrent with this recognition, there is a growing interest in the application of multiscale approaches (e.g., Coops and Waring, 2001; Zhang et al., 2002) for studying the critical zone. Such methodologies may help to identify typologies of system behavior that many scientists have anticipated to be highly complex, with chaotic characteristics at a fine scale of examination to more regulated or ordered and stable characteristics at larger scales (Svoraya and Shoshany, 2004).

The 18th World Congress of Soil Science took place in July 2006 in Philadelphia, PA, with the theme "Frontiers of Soil Science: Technology and the Information Age." The scientific program included topics on remote sensing, geographic information systems, landscape analysis, computer modeling, precision agriculture, and other applications of information science and technology as related to soils. A symposium held at the congress titled "Multiscale Mapping of Soil Properties for Environmental Studies, Agriculture, and Decision-Making" focused on techniques used in multiscale mapping of soil properties and processes. Papers covered theoretical and applied aspects of interpolation and extrapolation schemes, self-similar, hierarchal, and fractal organizations, spatial associations between variables,

S.D. Logsdon, USDA-ARS-NSTL, 2110 University Blvd., Ames, IA 50011; E. Perfect, Dep. of Earth and Planetary Sciences, Univ. of Tennessee, Knoxville, TN 37996-1410; A.M. Tarquis, Dep. de Matemática Aplicada a la Ingeniería Agronómica, E.T.S. Ingenieros Agrónomos, U.P.M., Spain. Received 26 Sept. 2007. Corresponding author (sally.logsdon@ars.usda.gov). 
and spatial-temporal dynamics. Selected papers from this symposium are combined with solicited papers on the same topic in this special section of the Vadose Zone Journal.

The purpose of this special section is to present cutting-edge tools and techniques, such as variograms and kriging, fractals and lacunarity, multifractal formalism, wavelets and spectral analysis, and entropy-based measures, used in multiscale mapping of soil properties and processes. Twelve papers are included, covering different quantitative techniques for data processing and analysis, assessment of prediction uncertainties, and integrative data analysis using combinations of field and laboratory data as well as simulated data sets. Linear, areal, and volumetric analyses are considered, and the range of scales covered runs from the poreparticle scale through peds, pedons, and soil associations up to the catena-watershed and regional scales.

Four of the papers use monofractal analyses in their multiscale investigations. Papadopoulos et al. (2008), working at the pore-particle scale, describe the use of the slit island method with image analysis to calculate fractal dimensions of soil pore perimeters. They show how differences in pore shape affect the scaling of pore perimeter measurements and propose an additional analysis coupled with the slit island method to capture the complexity of natural pore shapes and roughness. Blank et al. (2008) use methods derived from percolation theory to outline a numerical procedure to calculate for the hydraulic conductivity function from an arbitrary pore-size distribution. The approach extends critical path analysis to predict the hydraulic properties of porous media that do not exhibit monofractal scaling. Zamora-Castro et al. (2008) perform comparative monofractal and lacunarity analyses of layers from a soil monolith at four scanning electron microscopy image resolutions $(50 \times, 500 \times, 1000 x$, and 5000x). Lacunarity proved to be unique among the different parameters studied in its scale-dependent response to the multiscale image analyses. Tang et al. (2008) compute effective hydraulic properties for a monofractal sequence of layers of fine material interbedded within a coarse matrix. Their analytical model, based on the composite medium approximation applied to a Cantor bar, compares favorably with numerical simulations of unsaturated flow, based on Richards' equation, for small hydraulic gradients. Deviations between the two approaches increased as the hydraulic gradient increased, resulting in scale-dependent effective properties.

Multifractal analyses were applied to a wide variety of data, ranging from the pore through the field to the regional scale. Vidal Vázquez et al. (2008) analyze mercury injection porosimetry data collected on soil aggregates from surficial layers subjected to varying rainfall intensity. The entropy and correlation dimensions best discriminated between the resulting pore-size distributions. García Moreno et al. (2008) use multifractal formalism to characterize soil surface roughness created by three different tillage tools on sandy loam and sandy clay loam soils. They show that multifractal parameters, when combined into structural and complexity indices, provide better discrimination between the resulting surfaces than standard approaches based on the standard deviation and semivariogram. In another comparative study, Kravchenko (2008) examines the performance of stochastic simulations that reproduce multifractal scaling with simulations based on the traditional semivariogram. The multifractal method is shown to perform better than the semivariogram method for the prediction of low corn yields within an agricultural field. Cheng
(2008) presents a new multiscale mapping and data interpolation method based on multifractal analysis of an exploratory data set at the regional scale. Use of the technique is illustrated with geochemical data from 1172 lake sediments with the objective of identifying areas with strong singularities in concentration. Lovejoy et al. (2008) characterize the range of scaling of MODIS TERRA bands 1-7 using several methods such as spectra, trace moments, structure functions, and probability distribution multiple scaling. They show that these fields have strong resolution dependencies. Although vegetation and moisture indices derived from these bands can be correctly calibrated at a single resolution, they may be incorrect at other resolutions.

The final group of papers use diverse methodological approaches for investigating multiscale relationships. Si (2008) reviews spectra and wavelet methods, and associated scaling procedures, as applied to soil properties measured along linear transects across the landscape. A step-by-step description, as well as the advantages and disadvantages, is given of each procedure along with statistical testing of the different scaling techniques. Tarquis et al. (2008) describe a new entropy-based method, related to wavelets, for multiscale analysis of spatial data. The method was applied to soils data collected along a 1024-m transect to better understand the scaling behavior of soil water and gas fluxes and their scale-dependent correlations. Earls and Dixon (2008) use multiscale sensitivity analysis to investigate the vertical coupling between soil and atmospheric water fluxes and their relationship to potential evapotranspiration. They conduct a sensitivity analysis of Soil and Water Assessment Tool (SWAT) predicted potential evapotranspiration in response to multiscale meteorological input data and different calculation methods.

The 12 papers presented in this special section represent a range of highly original approaches for dealing with multiscale analysis of soil heterogeneity. The studies cover a wide range of scales, from microns to kilometers. In terms of future research, there is a need to extend the vast body of theory, methods, and applications for designing and optimizing soil sampling schemes to better incorporate information obtained at multiple scales. Spatial variability is often the outcome of nonlinear temporal fluctuations in multiple interacting soil-forming factors. This connection deserves further investigation. In addition, a clear distinction between systematic and random variations needs to be addressed in future studies. We hope that the research presented here will provide a stimulus for the development of more powerful analytical tools, as well as the collection of high-quality data sets in experiments specifically designed for multiscale analysis and hypothesis testing.

\section{References}

Blank, L.A., A.G. Hunt, and T.E. Skinner. 2008. A numerical procedure to calculate hydraulic conductivity for an arbitrary pore size distribution. Vadose Zone J. 7:461-472 (this issue).

Burrough, P.A. 1983. Multiscale sources of spatial variation in soil: I. The application of fractal concepts to nested levels of soil variation. Eur. J. Soil Sci. 34:577-597.

Burrough, P.A., J. Bouma, and S.R. Yates. 1994. The state of the art in pedometrics. Geoderma 62:311-326.

Cheng, Q. 2008. Modeling local scaling properties for multiscale mapping. Vadose Zone J. 7:525-532 (this issue).

Coops, N.C., and R.H. Waring. 2001. The use of multiscale remote sensing imagery to derive regional estimates of forest growth capacity using 3-PGS. Remote Sens. Environ. 75:324-334. 
Earls, J., and B. Dixon. 2008. A comparison of SWAT model-predicted potential evapotranspiration using real and modeled meteorological data. Vadose Zone J. 7:570-580 (this issue).

Eghball, B., G.W. Hergert, G.W. Lesoing, and R.B. Ferguson. 1999. Fractal analysis of spatial and temporal variability. Geoderma 88:349-362.

García Moreno, R., M.C. Díaz Álvarez, A. Saa Requejo, and A.M. Tarquis. 2008. Multifractal analysis of soil surface roughness. Vadose Zone J. 7:512-520 (this issue).

Heuvelink, G.B.M., and R. Webster. 2001. Modeling soil variation: Past, present, and future. Geoderma 100:269-301.

Hopmans, J.W., D.R. Nielsen, and K.L. Bristow. 2002. How useful are smallscale soil hydraulic property measurements for large-scale vadose zone modeling? p. 1-13. In D. Smiles P.A.C. Raats, and A. Warrick. (ed.) Heat and mass transfer in the natural environment, the Philip Volume. Geophysical Monogr. 129. AGU, Washington, DC.

Kravchenko, A.N. 2008. Stochastic simulations of spatial variability based on multifractal characteristics. Vadose Zone J. 7:521-524 (this issue).

Kravchenko, A.N., C.W. Boast, and D. Bullock. 1999. Multifractal analysis of soil spatial variability. Agron. J. 91:1033-1041.

Lin, H., J. Bouma, Y. Pachepsky, A. Western, J. Thompson, R. van Genuchten, H.-J. Vogel, and A. Lilly. 2006. Hydropedology: Synergistic integration of pedology and hydrology. Water Resour. Res. 42:W05301, doi:10.1029/ 2005WR004085.

Lovejoy, S., A.M. Tarquis, H. Gaonac'h, and D. Schertzer. 2008. Single- and multiscale remote sensing techniques, multifractals, and MODIS-derived vegetation and soil moisture. Vadose Zone J. 7:533-546 (this issue).

McBratney, A.B., M.L. Mendonca Santos, and B. Minasny. 2003. On digital soil mapping. Geoderma 117:3-52.

Pachepsky, Y., E. Perfect, and M.A. Martin. 2006. Fractal geometry applied to soil and related hierarchical systems. Geoderma 134:237-239.

Pachepsky, Y., D. Radcliffe, and H.M. Selim. 2003. Scaling methods in soil physics. CRC Press, Boca Raton, FL.

Papadopoulos, A., N.R.A. Bird, S.J. Mooney, and A.P. Whitmore. 2008. Fractal analysis of pore roughness in images of soil using the slit island method. Vadose Zone J. 7:456-460 (this issue).

Si, B.C. 2008. Spatial scaling analyses of soil physical properties: A review of spectral and wavelet methods. Vadose Zone J. 7:547-562 (this issue).

Sommer, M. 2006. Influence of soil pattern on matter transport in and from terrestrial biogeosystems: A new concept for landscape pedology. Geoderma 133:107-123.

Sposito, G. (ed.) 1998. Scale dependence and scale invariance in hydrology. Cambridge Univ. Press, Cambridge, UK.

Svoraya, T., and M. Shoshany. 2004. Multiscale analysis of intrinsic soil factors from SAR-based mapping of drying rates. Remote Sens. Environ. 92:233-246.

Tang, G., E. Perfect, E.H. van den Berg, M.A. Mayes, and J.C. Parker. 2008. Estimating effective hydraulic parameters of unsaturated layered sediments using a Cantor bar composite medium model. Vadose Zone J. 7:493-499 (this issue).

Tarquis, A.M., N.R. Bird, A.P. Whitmore, M.C. Cartagena, and Y. Pachepsky. 2008. Multiscale entropy-based analysis of soil transect data. Vadose Zone J. 7:563-569 (this issue).

Vereecken, H., R. Kasteel, J. Vanderborght, and T. Harter. 2007. Upscaling hydraulic properties and soil water flow processes in heterogeneous soils: A review. Vadose Zone J. 6:1-28.

Vidal Vázquez, E., J. Paz Ferreiro, J.G.V. Miranda, and A. Paz González. 2008. Multifractal analysis of pore size distributions as affected by simulated rainfall. Vadose Zone J. 7:500-511 (this issue).

Western, A.W., R.G. Grayson, and G. Bloschl. 2002. Scaling of soil moisture: A hydrologic perspective. Annu. Rev. Earth Planet. Sci. 30:149-180.

Zamora-Castro, S.A., K. Oleschko, L. Flores, E. Ventura, Jr., and J.F. Parrot. 2008. Fractal mapping of pore and solid attributes. Vadose Zone J. $7: 473-492$ (this issue).

Zhang, R., P. Shouse, S. Yates, and A. Kravchenko. 1997. Applications of geostatistics in soil science. Trends Soil Sci. 2:95-103.

Zhang, X.Y., N. Drake, and J. Wainwright. 2002. Scaling land surface parameters for global-scale soil erosion estimation. Water Resour. Res. 38:1180-1189. 